Pacific Journal of Mathematics

SOME SPECTRAL PROPERTIES OF POSITIVE LINEAR 


\title{
SOME SPECTRAL PROPERTIES OF POSITIVE LINEAR OPERATORS
}

\author{
Helmut SchaEFer
}

It is well known (Perron [12], Frobenius [6, 7]) that if $A$ is an $n \times n$ matrix over the real field with elements $\geqq 0$, the spectral radius ${ }^{1}$ of $A, r(A)$, is a characteristic number, with at least one characteristic vector whose coordinates are $\geqq 0$. If $A$ has positive elements throughout, then $r$ is $>0$, of algebraic and geometric multiplicity one, and exceeds all other elements of the spectrum in absolute value. ${ }^{2}$ Generalizations of this theorem to integral equations were obtained by Jentzsch [9] and E. Hopf [8]. In an operator-theoretic setting, the result did not appear until 1948 when Krein and Rutman published their most comprehensive work [11]. Further results were obtained by Bonsall [2]-[4] and, in the framework of a general locally convex space, by the author $[15,17]$ For compact positive operators in an order-complete Banach lattice, see Ando [1].

While the key to many results generalizing the Perron-Frobenius theorem is compactness in one form or another, a good many spectral properties of positive linear operators are independent of it. Such properties were established by Bonsall (e.c., cf. Prop. 1 below), the author [17], and recently Putnam [13] who considers, however, only the rather special case of a bounded matrix with non-negative elements in $l_{2}$. The present paper establishes new and more general results on the (spectral) character of the spectral radius $r$ of a positive operator $T$, valid in arbitrary ordered Banach spaces. ${ }^{3}$ Section 2 collects some theorems for which no hypothesis or $r$ is made; leaning heavily on topological properties of the positive cone $K$, they apply to any positive operator. Throughout $\S 3, r$ is assumed to be a pole of the resolvent of $T$. The stress is here on the notion of quasi-interior map; together with the assumption on $r$, this concept yields strong results earlier obtained by Krein and Rutman [11] for strongly positive operators ${ }^{17}$ which are compact and defined on a space whose positive cone $K$ has interior points. This is interesting since in many concrete examples of partially ordered $(B)$-spaces, $K$ has empty interior $[16, \mathrm{p} .130]$. The paper concludes with two problems.

Received October 20, 1959. Based on research sponsored by the Office of Ordnance Research, U.S. Army.

1 For the terminology adopted, see $\& 1$.

2 A short proof in [14]. Cf. also [5].

3 With only minor modifications, the results of the present paper carry over to bounded positive endomorphisms of a partially ordered, quasi-complete locally convex space. 
1. Auxiliary material. A (real or complex) Banach space $E$ is partially ordered if an order relation ${ }^{4}$, denoted $x \leqq y$ and invariant under addition and multiplication by positive scalars, is defined on $E$. It is well known that such an order structure is completely determined by the set $\{x: x \geqq 0\}$ of positive elements which will be called the positive cone $K$. Unless otherwise stated, we shall always suppose that $K$ is closed in $E$ and proper, i.e., such that $K \cap-K=\{0\}^{5} . \quad K$ is generating if $E=K-K$, normal if $\|x+y\| \geqq\|y\|$ for all $x, y \in K$ and some real norm $x \rightarrow\|x\|$ generating the topology of $E$. $K$ is a $B$-cone (BZKegel in [16]) if for some fundamental system of bounded sets $B$, the closed convex symmetric hulls of the sets $B \cap K, B \in \boldsymbol{B}$, form again a fundamental system of bounded subsets of $E .^{6}$ We say $K$ is spanned by a set $C$ if $K=\bigcup_{\lambda \geqq 0} \lambda C$. If $E^{\prime}$ is the topological dual of $E, K^{\prime} \subset E^{\prime}$ is the set of those linear forms which are $\geqq 0$ on $K$ (resp. if $E$ is complex, whose real parts are $\geqq 0$ on $K$ ). $K^{\prime}$ is called the cone conjugate to $K$. An $f \in E^{\prime}$ is positive (resp. strictly positive) with respect to a given partial ordering of $E$ if $\operatorname{Re} f(x) \geqq 0$ for $x \in K$ (resp. if $\operatorname{Re} f(x)>0$ for $0 \neq x \in K$ ). If $E$ is a real Banach space, $F$ its complexification in the usual sense, and $K$ is a normal cone (resp. a $B$-cone) in $E$, then $K+i K$ is a normal cone (resp. a $B$-cone) in $F[17$, p. 264].

Let $E$ denote a real or complex Banach space, partially ordered by a proper closed cone $K$.

Lemma 1. If $K$ is normal, then $E^{\prime}=K^{\prime}-K^{\prime}$. If $K$ is a normal $B$-cone, then so is $K^{\prime}$ for the strong topology on $E^{\prime}$.

The first part is proved (for real spaces) in [10]. For the second part, see [3, p. 146], and [17, p. 262/3] in the complex case. (It follows from a simple category argument that in a Banach space, every generating cone is a $B$-cone.)

An order interval in $E$ is a set $[x, y]=\{z: x \leqq z \leqq y\}$. We note that if $K$ is normal, every order interval is bounded.

DeFinition. A point $x$ is quasi-interior to $K$ if the order interval $[0, x]$ is a total subset of $E$.

It is clear that every interior point of $K$ is quasi-interior, and that every quasi-interior point of $K$ is a non-support point of $K$ in the sense of V. L. Klee. If $K$ has non-empty interior, the three notions coincide; this is the case, in particular, if $E$ is finite dimensional and $K$ is total (hence $K$, resp. $K+i K$ if $E$ is complex, is generating) in $E$.

4 i.e., a binary relation which is reflexive and transitive. We assume always that $E \neq\{0\}$.

${ }^{5} K$ is proper if and only if the order relation is anti-symmetric.

$6 S \subset E$ is symmetric if $x \in S$ implies $-x \in S$. In the (present) case of a normed space, $K$ is a $B$-cone if and only if there exists an $m>0$ such that every $x$ in the unit ball $U$ of $E$ is of the form $x=\lim _{n \rightarrow \infty}\left(u_{n}-v_{n}\right)$ with $u_{n}, v_{n} \in K \cap m U$. 
Lemma 2. Let $P$ be a continuous projection in $E$ such that $P K \subset K$. If $x \in P K$ is quasi-interior to $K$, it is quasi-interior to $P K$ in $P E$.

It is readily observed that $[0, x] \cap P E=P[0, x]$ under the conditions stated; since the linear hull of $[0, x]$ is dense in $E$, it follows that the linear hull of $P[0, x]$ is dense in $P E$.

A bounded endomorphism $T$ of $E$ is a positive operator if the positive cone $K$ is invariant under $T$, i.e., if $T K \subset K$. The spectral radius $r$ of $T$ is the maximum modulus of the points in its spectrum ${ }^{7} \sigma(T)$. The complement of $\sigma(T)$ in the complex plane is denoted by $\rho(T)$, and the resolvent $(\lambda-T)^{-1}$, locally holomorphic in $\rho(T)$, by $R_{\lambda}$. The point spectrum of $T$ is the set of all its characteristic numbers, i.e., the set of those $\lambda$ for which $\lambda-T$ fails to be $(1,1)$. For a characteristic number $\lambda, d(\lambda)$ denotes the (linear) dimension of the kernel of $\lambda-T$ (the characteristic space); an $x \neq 0$ in this kernel is called a characteristic vector (of $T$ for $\lambda$ ). It is well known that every pole of the resolvent is a characteristic number of $T$.

If $T$ is a positive operator, then so is its adjoint $T^{\prime}$ with respect to the conjugate cone $K^{\prime}$, which is a proper cone in $E^{\prime}$ if and only if $K$ is total in $E$.

DEFINITION. A positive operator $T$ is quasi-interior if there exists $\lambda>r$ ( $r$ the spectral radius of $T$ ) such that $T R_{\lambda} x$ is quasi-interior to $K$ for every $x, 0 \neq x \in K .^{8}$

This condition on $T$ is not stronger than requiring that for each $x, 0 \neq x \in K$, the union of order intervals $\bigcup_{n=1}^{\infty}\left[0, T^{n} x\right]$ be total in $E$. (It is clear that $K$ is a total cone in $E$ if the set of quasi-interior positive operators on $E$ is not empty.)

Lemma 3. If $K$ is a normal B-cone or, more generally, if $K$ and $K^{\prime}$ ( $K^{\prime}$ in the strong dual $\left.E^{\prime}\right)$ are normal cones, then the set $\Re$ of all positive operators is a normal cone in the Banach space $\mathcal{L}(E)$ of bounded endomorphisms of $E$.

It is known [17, p. 269] that the assertion holds if $K$ is a normal $B$-cone in $E$. If $K$ and $K^{\prime}$ are both normal, then $K^{\prime}$ is a normal $B$-cone for the strong topology on $E^{\prime}$ (this follows from Lemma 1 and the subsequent remark); therefore by Lemma 1 , the cone $K^{\prime \prime}$ conjugate to $K^{\prime}$ in the Banach space $E^{\prime \prime}$, bidual of $E$, is a normal $B$-cone. Thus the cone $\Omega^{\prime \prime}$ of positive operators on $E^{\prime \prime}$ (with respect to $K^{\prime \prime}$ ) is normal

7 If $E$ is a real space, the terms spectrum, resolvent etc. will be understood with respect to the extension of $T$ to the complexification of $E$, which may be considered as ordered with positive cone $K$ or $K+i K$.

8 E.g., if $E=l_{2}, K$ the cone of all vectors with non-negative coordinates, a bounded matrix $A=\left(a_{i, k}\right)$ with non-negative elements is quasi-interior if and only if for each pair $(i, k)$ of indices, there exists $n=n(i, k)$ such that $\left(A^{n}\right)_{i, k}>0$. Cf. [13]. 
in $\mathcal{L}\left(E^{\prime \prime}\right)$ and this implies that $\Omega$ is normal in $\mathcal{L}(E)$ because the normpreserving natural imbedding of $\mathcal{L}(E)$ into $\mathcal{L}\left(E^{\prime \prime}\right)$ maps $\Omega$ into $\Omega^{\prime \prime}$.

2. Some properties of the spectral radius. Throughout this section, $E$ denotes a (real or complex) partially ordered Banach space with positive cone $K ; E^{\prime}$ is the (topological) dual of $E$, equipped with the strong topology unless otherwise stated. $T$ is a positive operator on $E$ with spectral radius $r$.

The first part of the following proposition is due to Bonsall [3, p. 148] but the proof given here, which also yields the second assertion, is entirely different from that in [3].

Proposition 1. Let $K$ and $K^{\prime}$ be normal cones in $E$ resp. $E^{\prime}$. For each positive operator $T, r$ is in the spectrum of $T$. If $r$ is a pole of the resolvent $R_{\lambda}$ of order $k$, every other pole of $R_{\lambda}$ on $|\lambda|=r$ is of an order $\leqq k$.

Proof. It follows from Lemma 3 that the cone $\Omega$ of positive operators is normal in $\mathfrak{Q}(E)$ with respect to the uniform topology. It is shown in [18] that if $z \rightarrow f(z)$ is an analytic function with values in a Banach space, holomorphic at 0 , such that its expansion at $0, \sum_{n=0}^{\infty} \alpha_{n} z^{n}$, has radius of convergence 1 and the set of coefficients $\left\{a_{n}\right\}$ is contained in a normal cone, then $z=1$ is singular for $f$ and if it is a pole of order $k$, there is no pole of $f$ on $|z|=1$ of order $>k$. The proposition follows immediately by letting $f(z)=R(r / z)$ if $r>0\left(R_{\lambda}=R(\lambda)\right.$ the resolvent of $T$ ). If $r=0$, the result is trivial.

Proposition 2. $R_{\lambda}$ is a positive operator for each (real) $\lambda>r$; if $R_{\lambda}$ is positive for some $\lambda \in \rho(T)$, then $\lambda$ is real and $>0 .^{9}$ If $K, K^{\prime}$ are normal (hence, if $K$ is a normal $B$-cone), then $\lambda>r$ is a necessary and sufficient condition in order that $R_{\lambda}$ be positive.

Proof. From the expansion of $R_{\lambda}$ at $\infty$, it is easily seen that the condition $\lambda>r$ is sufficient. Now assume that for some $\lambda \in \rho(T), R_{\lambda}$ is a positive operator. Select an $x_{0} \in K, x_{0} \neq 0$, and define recursively $x_{n}=R_{\lambda} x_{n-1}(n \in N) .^{10}$ Each $x_{n}$ satisfies the equation

$$
\lambda x_{n}=T x_{n}+x_{n-1} .
$$

We have $x_{n} \in K(n \in N)$ and since $x_{n}=0$ for some $n$ would imply $x_{0}=0, x_{n} \neq 0$ for all $n$. From $\left(^{*}\right)$ it follows that $\lambda x_{1} \in K$, and by induction it is established that $\lambda^{n} x_{n} \in K, \lambda^{n-1} x_{n} \in K$ for all $n \in N$. Also,

${ }^{9}$ For this statement, we have to assume that $K \neq\{0\}$.

${ }^{10} \mathrm{~N}$ stands for the set of positive integers. 


$$
\lambda^{n} x_{n} \geqq \lambda^{n-1} x_{n-1} \geqq x_{0}
$$

Thus $\lambda \neq 0$ and without loss of generality, we may assume that $|\lambda|=$ 1. (For if $R_{\lambda}$ is positive at $\lambda \neq 0$, then the resolvent of $\left|\lambda^{-1}\right| T$ is positive at $\lambda\left|\lambda^{-1}\right|$.) Let $\lambda=e^{i \varphi}, 0 \leqq \varphi<2 \pi$, and suppose that $\phi>0$. It is clear that $n \varphi \neq \pi(n \in N)$ or $K$ would not be a proper cone. Hence there is an $n_{0} \in N$ such that the triangle in the complex plane with vertices $1, e^{i\left(n_{0}-1\right) \varphi}, e^{i n_{0} \varphi}$ contains 0 in its interior. Consider the 2-dimensional real subspace $L$ of $E$ (resp. of $E+i E)^{7}$ containing $x_{n_{0}}$ and $i x_{n_{0}}$. $K \cap L$ (resp. $\left.(K+i K) \cap L\right)$ is a proper convex cone of vertex 0 in $L$ containing the points $x_{n_{0}}, \lambda^{n_{0}-1} x_{n_{0}}, \lambda^{n_{0}} x_{n_{0}}$. Hence this cone contains 0 as an interior point in $L$ which is contradictory. Thus $\varphi=0$, and $\lambda>0$.

Let $K$ and $K^{\prime}$ be normal in $E$ resp. $E^{\prime}$; then the cone $\Re$ of positive operators is normal in $\mathfrak{Q}(E)$ by Lemma 3 . If we had $R_{\lambda} \in \Omega$ for some $\lambda, 0<\lambda<r$, from the resolvent equation

$$
R_{\lambda}-R_{\mu}=(\mu-\lambda) R_{\lambda} R_{\mu}
$$

it would follow that $R_{\mu} \leqq R_{\lambda}$ (with respect to the order relation on $\mathfrak{L}(E)$ whose positive cone is $\Re)$ for all $\mu>\lambda$, for which $R_{\mu} \geqq 0$ therefore, in particular, for all $\mu>r$. This would imply $\left\|R_{\mu}\right\| \leqq\left\|R_{\lambda}\right\|$ for all $\mu>r$ and some real norm $A \rightarrow\|A\|$ generating the topology of bounded convergence on $\mathfrak{L}(E)$. This is impossible since $r \in \sigma(T)$ by Prop. 1 and consequently, $\left\|R_{\mu}\right\| \rightarrow \infty$ as $\mu \downarrow r$. The proof is finished.

Proposition 3. If there exists $y, 0 \neq y \in K$, such that $T^{p} y \geqq \delta y$ for some $p \in N$ and $\delta>0$, then $r \geqq \delta^{1 / p}$.

Proof. Since $K$ is closed and $\neq E$, a routine argument shows that there exists a continuous linear form $h \in E^{\prime}$ such that the real part $f(x)=\operatorname{Re} h(x)$ is $\geqq 0$ on $K$ and $f(y)>0$. For $\lambda>r$, we have

$f\left(R_{\lambda} y\right)=\sum_{n=0}^{\infty} \frac{1}{\lambda^{n+1}} f\left(T^{n} y\right) \geqq \sum_{k=1}^{\infty} \frac{1}{\lambda^{k p+1}} f\left(T^{k p} y\right) \geqq f(y) \sum_{k=1}^{\infty} \frac{\delta^{k}}{\lambda^{k p+1}}=f(y) \cdot \frac{\delta}{\lambda\left(\lambda^{p}-\delta\right)}$

because $T^{p} y \geqq \delta y$ implies $T^{k p} y \geqq \delta^{k} y(k \in N)$. It follows that $f\left(R_{\lambda} y\right)$ is unbounded as $\lambda^{p} \downarrow \delta$. Consequently $r \geqq \delta^{1 / p}$.

THEOREM 1. Let $K$ be spanned by a convex set not containing 0 and compact for some locally convex topology (on $E$ ) for which $T$ is continuous on $K^{11}$. There exists a non-negative characteristic number

11 i.e., for which the restriction of $T$ to $K$ is continuous. 
of $T$ with (at least one) characteristic vector in $K$. If in addition $K$ is a normal cone generating $E$, then $r$ is such a number. ${ }^{12}$

Proof. Let $C$ be the convex set and $\mathfrak{I}$ the locally convex topology in question. There exists a $\mathfrak{I}$-closed real hyperplane $H=\{x: f(x)=1\}$ separating $C$ strictly from 0 . It is clear that $f(x)>0$ for $0 \neq x \in K$. $K$ is closed for $\mathfrak{T}$ : Let $\boldsymbol{F}$ be a filter on $K$ converging to $x_{0} \in E$ for $\mathfrak{T}$; since $f$ is continuous, there exists $F \in F$ such that $\sup \{f(x): x \in F\} \leqq$ $1+f\left(x_{0}\right)$, therefore $F \subset\left(1+f\left(x_{0}\right)\right) C_{1}$, where $C_{1}$ is the convex hull of $\{0\}$ and $C$. Since $C_{1}$ is compact ${ }^{13}, x_{0}$ which is in the closure of $F$, is in $K$. Because $H \cap K$ is a closed subset of $C_{1}, H \cap K$ is compact; so $f\left(x_{n}\right) \rightarrow 0$ implies $x_{n} \rightarrow 0$ and thus $T x_{n} \rightarrow 0$ for any sequence $\left\{x_{n}\right\} \subset K$, (all statements in this sentence referring to $\mathfrak{I}$ ).

Consider the real subspace $\hat{E}=K-K$ of $E$, equipped with the norm

$$
z \rightarrow\|z\|=\inf \{f(x)+f(y): z=x-y ; x, y \in K\} .
$$

$\hat{E}$ is a Banach space. Given an arbitrary Cauchy sequence in $\hat{E}$, there exists a subsequence $\left\{z_{k}\right\}$ such that $\left\|z_{k+1}-z_{k}\right\|<1 / 2^{k}$. By definition of the norm in $\hat{E}$, there exist two sequences $\left\{x_{k}\right\},\left\{y_{k}\right\}$ in $K$ with $z_{k+1}-z_{k}=x_{k}-y_{k}(k \in N)$ and $\left\|x_{k}\right\|+\left\|y_{k}\right\| \leqq 1 / 2^{k}$. Since $C_{1}$ is compact for $\mathfrak{I}$, the sequence

$$
\left\{\sum_{\nu=1}^{n} x_{\nu}: n \in N\right\}\left(\operatorname{resp} \cdot\left\{\sum_{\nu=1}^{n} y_{\nu}: n \in N\right\}\right)
$$

has a limit point $x$ (resp. $y$ ) in $K$, and it is now easy to see that $\left\{z_{k}\right\}$ (and hence the given sequence) converges to $x-y$, in $\hat{E}$. It is readily verified that the restriction $\hat{T}$ of $T$ to $\hat{E}$ is a continuous endomorphism. Moreover, $K$ is a normal closed cone in $\hat{E}$, and it is a $B$-cone since it is generating (cf. the remark following Lemma 1). If $\hat{r}$ is the spectral radius of $\hat{T}$, we have $\hat{r} \in \sigma(\hat{T})$ by Prop. 1. Thus, since $\hat{R}_{\lambda} x$ is nondecreasing for each $x \in K$ if $\lambda \downarrow \hat{r}$, we have $\left\|\hat{R}_{\lambda} y\right\| \rightarrow \infty$ for some $y \in K$ as $\lambda \downarrow \hat{r}$. Let $\lambda_{n} \downarrow \hat{r}$ and set $x_{n}=\hat{R}\left(\lambda_{n}\right) y /\left\|\hat{R}\left(\lambda_{n}\right) y\right\|$. Then $\lambda_{n} x_{n}-\hat{T} x_{n} \rightarrow 0$ in $\hat{E}$ and also $(\hat{r}-\hat{T}) x_{n} \rightarrow 0$ because of $\left\|x_{n}\right\|=1$. By Proposition 2, $x_{n} \in K$; and, since $1=\left\|x_{n}\right\|=f\left(x_{n}\right)$, it follows that $x_{n} \in H \cap K(n \in N)$. Now $H \cap K$ is compact for $\mathfrak{I}$ and as $\hat{r}-\hat{T}$ is continuous for $\mathfrak{I}$ on $K$, it follows that $(\hat{r}-\hat{T}) x=0$ for some $x \in H \cap K$. The proof of the first part is finished.

12 The assumption that $K$ be closed in $E$ is not needed in Th. 1 and the corollary; the first assertion of Th. 1 is also independent of $E$ being a Banach space and of $T$ being bounded.

${ }^{13}$ In any linear topological space, the convex hull of a finite number of convex compact sets is compact. A locally convex topology is assumed to be Hausdorff by definition. 
If $K$ is a normal generating cone in $E$, then $r \in \sigma(T)$ by Prop. 1 . It is clear that $\hat{r} \leqq r$. On the other hand, $\hat{r}<r$ would imply that $r-T$ is an algebraical automorphism of $E$, which is impossible.

REMARK. Using the notation of the preceding proof, the number $\hat{r}$ (which was shown to be in the point spectrum of $T$ ) may be characterized as follows:

(a) $\hat{r}$ is the greatest real number $\alpha$ such that $\alpha-T$ is not an algebraical automorphism of the real subspace $K-K$ of $E$.

(b) $\hat{r}$ is the smallest real number $\alpha$ such that $R_{\lambda}$ is positive for $\lambda>\alpha$, $\lambda \in \rho(T)$.

(c) If $g$ is a real $\mathfrak{I}$-continuous linear form on $E$ with $0 \notin g(C)$, then

$$
\hat{r}=\lim _{n \rightarrow \infty}\left\{\sup \left|g\left(T^{n} x\right)\right|: x \in C\right\}^{1 / n} .
$$

As an application of Th. 1, we list a proposition which is equivalent to the combination of [2, Th. 1] and [4, Th. C].

COROLlaRY. If $K$ has non-empty interior, there exists a non-negative number in $\sigma(T)$ which is a characteristic number of $T^{\prime}$ with (at least one) characteristic vector in $K^{\prime}$. If in addition $K$ is normal, then $r$ is such a number.

Proof. If $x_{0}$ is interior to $K$, the real hyperplane $H=\left\{x^{\prime} \in E^{\prime}\right.$ : $\left.\operatorname{Re}\left\langle x^{\prime}, x_{0}\right\rangle=1\right\}$ intersects $K^{\prime}$ is a set compact for the weak* topology on $E^{\prime}$. For the linear forms in this intersection are uniformly bounded on the order interval $\left[0, x_{0}\right]$ (which has interior points), hence equicontinuous. Obviously $H \cap K^{\prime}$ spans $K^{\prime}$, and $T^{\prime}$ is continuous for the weak ${ }^{*}$ topology. The assertion concerning $T$ follows from $\sigma(T)=\sigma\left(T^{\prime}\right)$. Finally, if in addition $K$ is normal, $K^{\prime}$ is a normal $(B)$-cone in $E^{\prime}$ spanning $E^{\prime}$ by Lemma 1 which completes the proof.

REMARK. If $K$ is normal with non-empty interior $\stackrel{\circ}{K}$, then for each $x_{0} \in \stackrel{\circ}{K}$, the norm $A \rightarrow\|A\|_{x_{0}}=\sup \left\{\|A x\|: x \in\left[0, x_{0}\right]\right\}$ generates the topology of bounded convergence on $\mathfrak{L}(E)$. For a positive operator and a norm on $E$ which is monotone on $K,\|T\|_{x_{0}}=\left\|T x_{0}\right\|$. Thus:

If $K$ is normal with $\stackrel{\circ}{K} \neq \phi$ (and $T$ positive), then

$$
r=\lim _{n \rightarrow \infty}\left\|T^{n} x_{0}\right\|^{1 / n}
$$

for every $x_{0} \in \stackrel{\circ}{K}$.

3. Operators for which $r$ is a pole of $R_{\lambda}$. As in $\S 2, E$ denotes a (real or complex) partially ordered Banach space; but we shall assume 
that $T$ is a positive operator for which the spectral radius $r$ is a pole of the resolvent $R_{\lambda}$. The positive cone $K$ is assumed proper and closed.

Proposition 4. The leading coefficient in the principal part of $R_{\lambda}$ at $\lambda=r$ is a positive operator. Hence, if $K$ is total in $E$, there exists (at least) one characteristic vector of $T$ for $r$ in $K$, and of $T^{\prime}$ for $r$ in $K^{\prime}$.

Proof. Since the leading coefficient in the principal part of $R_{\lambda}$ is the $\operatorname{limit}^{14}$ ( $r$ being a pole of order $k$ ) of $(\lambda-r)^{k} R_{\lambda}$ as $\lambda \downarrow r$, the first assertion follows from the facts that $R_{\lambda}$ is positive for $\lambda>r$ and that $K$ is closed in $E$. Further, if $K$ is a closed proper cone total in $E$, then $K^{\prime}$ is a closed proper cone weak* total in $E^{\prime}$. The remainder is clear.

Theorem 2. Let $T$ be quasi-interior. Then:

$1^{\circ}$. $\quad r>0$ and $r$ is a simple pole of $R_{\lambda}$.

$2^{\circ}$. Every characteristic vector pertaining to $r$, of $T$ in $K$ (resp. of $T^{\prime}$ in $K^{\prime}$ ) is quasi-interior to $K$ (resp. a strictly positive linear form).

$3^{\circ}$. Each of these conditions implies that $d(r)=1$ :

(a) $K$ has non-empty interior

(b) $d(r)$ is finite

(c) $E$ is a Banach lattice..$^{15}$

Proof. The assumption $r=0$ implies, by Prop. 4, that $T x=0$ for some $x, 0 \neq x \in K$. (Since $T$ is a quasi-interior map, $K$ has quasi-interior points and is therefore total in $E$.) But then $T R_{\lambda} x=0$ for every $\lambda \in \rho(T)$ which contradicts the definition of a quasi-interior map. Hence $r>0$.

Let $x_{0}, 0 \neq x_{0} \in K$, be a characteristic vector of $T$ for $r$. By definition, there exists $\lambda>r$ such that $T R_{\lambda} x_{0}$ is quasi-interior to $K$. From

$$
T R_{\lambda} x_{0}=\sum_{1}^{\infty} \frac{1}{\lambda^{n}} T^{n} x_{0}=x_{0} \sum_{1}^{\infty}\left(\frac{r}{\lambda}\right)^{n}
$$

it follows that $x_{0}$ is quasi-interior to $K$. Similarly, if $f$ is a characteristic vector of $T^{\prime}$ in $K^{\prime}$ for $r$, we have $r^{n} f(x)=f\left(T^{n} x\right)(n \in N)$ for $x \in E$, hence with $f_{1}(x)=\operatorname{Re} f(x)$

$$
f_{1}(x) \sum_{1}^{\infty}\left(\frac{r}{\lambda}\right)^{n}=\sum_{1}^{\infty} \frac{1}{\lambda^{n}} f_{1}\left(T^{n} x\right)=f_{1}\left(T R_{\lambda} x\right)>0
$$

14 For the topology of bounded convergence.

15 In the sense of G. Birkhoff (Lattice Theory, New York 1948). A Banach lattice is by definition a real space; for our purposes, it is sufficient to assume that the underlying real space of $E$ is a Banach lattice. 
for every $0 \neq x \in K$, for $f_{1}$ must be $>0$ at every quasi-interior point of $K$.

We show that $r$ is a simple pole of $R_{\lambda}$. Let $k$ be the order of $r$; if $A$ is the leading coefficient in the principal part of $R_{\lambda}$ at $\lambda=r$, we have $A=P(T-r)^{k-1}$ where

$$
P=\frac{1}{2 \pi i} \int_{0} R_{\lambda} d \lambda
$$

( $C$ a positively oriented circle enclosing $r$, and having no other elements of $\sigma(T)$ in its interior or on its boundary), is the continuous projection of $E$ onto the subspace pertaining to the spectral set $\{r\} . K$ being total in $E$, we have $A v \neq 0$ for some $v \in K$ and $A v$ is quasi-interior to $K$ by $2^{\circ}$. Let $f \in K^{\prime}$ be a characteristic vector of $T^{\prime}$ for $r$ (Prop. 4), then $P^{\prime} f=f\left(P^{\prime}\right.$ the adjoint of $\left.P\right)$ and

$$
\left.\left.f_{1}(A v)=f_{1} \mid(T-r)^{k-1} v\right]=\mid\left(T^{\prime}-r\right)^{k-1} f\right\rfloor_{1}(v)>0
$$

which implies $k=1$. Therefore, $r$ is a simple pole.

We show now that $3^{\circ}$. holds. Since $r$ is a simple pole of $R_{\lambda}, P$ is a positive operator by Prop. 4 . If $x_{0} \in K$ is a characteristic vector of $T$ for $r, x_{0}$ is quasi-interior to $K$ by $2^{\circ}$. Therefore, the cone $P K$ can have no boundary points $\neq 0$ which are not quasi-interior to $P K$ in $P E$ by Lemma 2 . If a) $K$ has interior points, then so has $P K$ in $P E$; thus we must have $d(r)=1$. If $b) d(r)$ is finite, i.e., if $P$ is of finite rank, then every quasi-interior point of $P K$ is actually interior to $P K$ in $P E$ and the conclusion is the same.

There remains to show that $3^{\circ}$. c) is sufficient for $d(r)=1$. Let $x_{0}$ be any characteristic vector of $T$ for $r$. We have $r x_{0}=T x_{0}$ and consequently $r\left|x_{0}\right| \leqq T\left|x_{0}\right|,\left|x_{0}\right|$ denoting the absolute of $x_{0}$ in the latticetheoretic sense. If in the latter relation equality does not hold, we obtain

$$
r f_{1}\left(\left|x_{0}\right|\right)<f_{1}\left(T\left|x_{0}\right|\right)=r f_{1}\left(\left|x_{0}\right|\right)
$$

for every characteristic vector $f \in K^{\prime}$ of $T^{\prime}$ for $r$ ( $f$ is then strictly positive by $2^{\circ}$ ). This is contradictory; hence, $r\left|x_{0}\right|=T\left|x_{0}\right|$ for every characteristic vector $x_{0}$, whether or not in $K$, of $T$ for $r$. Now $x_{0}=$ $x_{0}^{+}-x_{0}^{--}$where the summands are disjoint. Since $\left|x_{0}\right|=x_{0}^{+}+x_{0}^{-}, x_{0}^{+}$and $x_{0}^{-}$are both in the characteristic space of $T$ pertaining to $r$. Assume that for some $x_{0}$, both $x_{0}^{+} \neq 0$ and $x_{0}^{-} \neq 0$. Since the order interval $\left[0, x_{0}^{+}\right]$is disjoint from $x_{0}^{-}$and the lattice operations are continuous, $x_{0}^{+}$ cannot be quasi-interior to $K$ which contradicts $2^{\circ} \cdot{ }^{16}$ Consequently, either

${ }^{16}$ It becomes clear from this that if $E$ is a Banach lattice, the points quasi-interior to $K$ are weak units of $E$ in the sense of Birkhoff (1.c.). 
$x_{0}^{+}=0$ or $x_{0}^{-}=0$. This implies that for each characteristic vector of $T$ in $K$ (for $r$ ), either $x_{0} \in K$ or $x_{0} \in-K$; therefore $d(r)=1$.

The theorem is proved.

If the assumptions that $T$ be quasi-interior and $r$ be a pole of $R_{\lambda}$ are satisfied, $r$ need not be the only element of $\sigma(T)$ on $|\lambda|=r$ even if $E$ is finite dimensional. For let $E$ be Euclidean 2-space in its natural order (i.e., $K$ being the set of all vectors with non-negative coordinates). The positive operator on $E$ represented by the matrix $\left(\begin{array}{ll}0 & 1 \\ 1 & 0\end{array}\right)$ is quasiinterior: for $\lambda=2, R_{\lambda}$ is the matrix $1 / 3\left(\begin{array}{ll}2 & 1 \\ 1 & 2\end{array}\right)$. The characteristic numbers of $T$ are 1 and -1 .

Proposition 5. Let $T$ be such that for each $x, 0 \neq x \in K$, there exists a positive integer $n=n(x)$ for which $T^{n} x$ is an interior point of $K{ }^{17} \quad$ Then $r$ is the only element in the point spectrum of $T$ on $|\lambda|=r$.

Proof. We note first that if $T$ has the stated property and $E$ is a real space, the extension of $T$ to the complexification $E+i E$ has the same property provided $E+i E$ is considered as partially ordered with positive cone $K+i K$. Hence we assume $E$ as complex.

By Theorem 2 (since $T$ is obviously quasi-interior) there exists $x_{0}$ interior to $K$ with $r x_{0}=T x_{0}$. Because of $r>0$, we may assume that $r=1$. Suppose that for some $\varphi, 0<\varphi<2 \pi, e^{i \varphi}$ is in the point spectrum of $T$ and $e^{i \varphi} x=T x(x \neq 0)$. Consider the 3 -dimensional real subspace $E_{3}$ of $E$ that contains $x_{0}, x, i x$; obviously $E_{3}$ is invariant under $T$. $x_{0}$, which is an interior point of $K$, is interior to $K_{3}=K \cap E_{3}$ in $E_{3}$. Identifying $E_{3}$ (which we may for our purpose) as Euclidean 3-space with coordinate axes $x_{0}, x, i x$, the restriction of $T$ to $E_{3}$ is a rotation through $\varphi$ about $x_{0}$. Let $w \neq 0$ be a point of $K_{3}$ which has maximum angular distance from $x_{0}$; then $T^{n} w$ must have the same property for every $n \in N$. This implies that no $T^{n} w(n \in N)$ is interior to $K_{3}$ and a contradiction is established.

4. Problems. Let $E$ be a partially ordered Banach space with positive cone $K, T$ a positive operator on $E$ with spectral radius $r$. Under what general conditions, if any, are these implications true:

a. If $r$ is an isolated singularity of $R_{\lambda}$, every singularity of $R_{\lambda}$ on $|\lambda|=r$ is isolated.

b. If $r$ is a pole of $R_{\lambda}, R_{\lambda}$ has no singularities on $|\lambda|=r$ other than poles. ${ }^{18}$

17 Operators $T$ with this property are called strongly positive in [11].

18 E.g., are a. and b. true if $K$ is a normal $B$-cone in $E$ ? 


\section{REFERENCES}

1. T. Ando, Positive linear operators in semi-ordered linear spaces, J. Fac. Science, Hokkaido University, Ser. I, XIII, (1957), 214-228.

2. F. F. Bonsall, Endomorphisms of partially ordered vector spaces, J. London Math. Society 30, (1955), 133-144.

3. Lndomorphisms of a partially ordered vector space without order unit, ibidem, 144-153.

4. L Linear operators in complete positive cones, Proc. London Math. Soc. 3, VIII (29), (1958), 53-75.

5. A. Brauer, A new proof of theorems of Perron and Frobenius on non-negative matrices, I. Positive matrices. Duke Math. J., 24, (1957), 367-378.

6. G. Frobenius, Uber Matrizen aus positiven Elementen, Sitz. Ber. Preuss. Akademie der Wiss. Berlin. (1908), 471-476. (1909), 514-518.

7. — Uber Matrizen aus nicht negativen Elementen, ibidem (1912), 456-477.

8. E. Hopf, Uber lineare Integralgleichungen mit positivem Kern, Sitz. Ber. Preuss. Akademie der Wiss. Berlin. XVIII, (1928), 233-245.

9. R. Jentzsch, Uber Integralgleichungen mit positivem Kern, Crelles J. 141, (1912), 235-244. 10. M.G. Krein, et J. Grosberg, Sur la décomposition des fonctionnelles en composantes positives, Dokl. Acad. Sci. U.R.S.S. (N.S.) 25 (1939), 723-726.

11. M. G. Krein, Linear operators leaving invariant a cone in a Banach space, Uspehi Mat. Nauk. (n.s.) 3, no. 1 (23), (1948), 3-95. Amer. Math. Soc. Transl. No. 26.

12. O. Perron, Zur Theorie der Matrizes, Math. Ann. 64, (1907), 248-263.

13. C. R. Putnam, On bounded matrices with non-negative elements, Canad. J. Math. X(4), (1958), 587-591.

14. H. Samelson, On the Perron-Frobenius theorem, Michigan Math. J., (1957), 57-59.

15. H. Schaefer, Positive Transformationen in lokalkonvexen halbgeordneten Velktorräumen, Math. Ann. 129, (1955), 323-329.

16. — Halbgeordnete lokalkonvexe Vektorräume, Math. Ann. 135, (1958), 115-141.

17. - Halbgeordnete lokalkonvexe Vektorräume, II Math. Ann. 138, (1959), 259-286.

18. - On the singularities of an analytic function with values in a Banach space, Arch. Math. XI, (1960), 40-43.

UNIVERSITY OF MICHIGAN 



\section{PACIFIC JOURNAL OF MATHEMATICS}

\section{EDITORS}

\author{
David Gilbarg \\ Stanford University \\ Stanford, California \\ F. H. Brownell \\ University of Washington \\ Seattle 5 , Washington
}

\author{
A. L. Whiteman \\ University of Southern California \\ Los Angeles 7, California \\ L. J. PAIGE \\ University of California \\ Los Angeles 24, California
}

\section{ASSOCIATE EDITORS}

\author{
E. F. BECKENBACH \\ T. M. CHERRY \\ D. DERRY
}
E. HEWITT
A. HORN
L. NACHBIN
M. OHTSUKA
H. L. ROYDEN
M. M. SCHIFFER

E. SPANIER

E. G. STRAUS

F. WOLF

\section{SUPPORTING INSTITUTIONS}

\author{
UNIVERSITY OF BRITISH COLUMBIA \\ CALIFORNIA INSTITUTE OF TECHNOLOGY \\ UNIVERSITY OF CALIFORNIA \\ MONTANA STATE UNIVERSITY \\ UNIVERSITY OF NEVADA \\ NEW MEXICO STATE UNIVERSITY \\ OREGON STATE COLLEGE \\ UNIVERSITY OF OREGON \\ OSAKA UNIVERSITY \\ UNIVERSITY OF SOUTHERN CALIFORNIA
}

\author{
STANFORD UNIVERSITY \\ UNIVERSITY OF TOKYO \\ UNIVERSITY OF UTAH \\ WASHINGTON STATE COLLEGE \\ UNIVERSITY OF WASHINGTON

AMERICAN MATHEMATICAL SOCIETY
CALIFORNIA RESEARCH CORPORATION
HUGHES AIRCRAFT COMPANY
SPACE TECHNOLOGY LABORATORIES
NAVAL ORDNANCE TEST STATION

Mathematical papers intended for publication in the Pacific Journal of Mathematics should be typewritten (double spaced), and the author should keep a complete copy. Manuscripts may be sent to any one of the four editors. All other communications to the editors should be addressed to the managing editor, L. J. Paige at the University of California, Los Angeles 24, California. ..

50 reprints per author of each article are furnished free of charge; additional copies may be obtained at cost in multiples of 50 .

The Pacific Journal of Mathematics is published quarterly, in March, June, September, and December. The price per volume (4 numbers) is $\$ 12.00$; single issues, $\$ 3.50$. Back numbers are available. Special price to individual faculty members of supporting institutions and to individual members of the American Mathematical Society: $\$ 4.00$ per volume; single issues, $\$ 1.25$.

Subscriptions, orders for back numbers, and changes of address should be sent to Pacific Journal of Mathematics, 2120 Oxford Street, Berkeley 4, California.

Printed at Kokusai Bunken Insatsusha (International Academic Printing Co., Ltd.), No. $6_{4}$ 2-chome, Fujimi-cho, Chiyoda-ku, Tokyo, Japan.

PUBLISHED BY PACIFIC JOURNAL OF MATHEMATICS, A NON-PROFIT CORPORATION

The Supporting Institutions listed above contribute to the cost of publication of this Journăl, but they are not owners or publishers and have no responsibility for its content or policies. 


\section{Pacific Journal of Mathematics}

\section{Vol. 10, No. $3 \quad$ November, 1960}

Glen Earl Baxter, An analytic problem whose solution follows from a simple

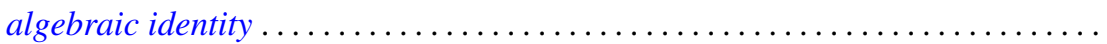

Leonard D. Berkovitz and Melvin Dresher, A multimove infinite game with linear payoff. .

Earl Robert Berkson, Sequel to a paper of A. E. Taylor ......................

Gerald Berman and Robert Jerome Silverman, Embedding of algebraic systems.... 767

Peter Crawley, Lattices whose congruences form a boolean algebra . . . . . ...... 777

Robert E. Edwards, Integral bases in inductive limit spaces . . . . . . . . . . . . . . .

Daniel T. Finkbeiner, II, Irreducible congruence relations on lattices . . . . . . . . . .

William James Firey, Isoperimetric ratios of Reuleaux polygons . . . . . . . . . . . 787

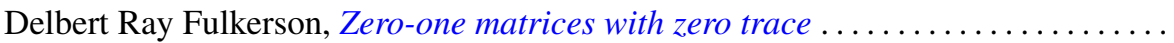

Leon W. Green, A sphere characterization related to Blaschke's conjecture........

Israel (Yitzchak) Nathan Herstein and Erwin Kleinfeld, Lie mappings in

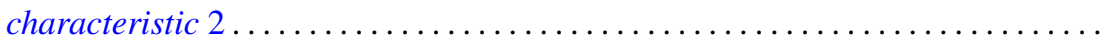

Charles Ray Hobby, A characteristic subgroup of a $p$-group .................

R. K. Juberg, On the Dirichlet problem for certain higher order parabolic

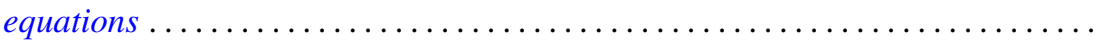

Melvin Katz, Infinitely repeatable games ......................

Emma Lehmer, On Jacobi functions . . . . . . . . . . . . . . . . . . . . . . . . .

D. H. Lehmer, Power character matrices

Henry B. Mann, A refinement of the fundamental theorem on the density of the sum of two sets of integers.

Marvin David Marcus and Roy Westwick, Linear maps on skew symmetric matrices: the invariance of elementary symmetric functions . .

Richard Dean Mayer and Richard Scott Pierce, Boolean algebras with ordered

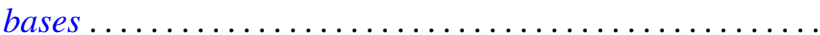

Trevor James McMinn, On the line segments of a convex surface in $E_{3} \ldots$

Frank Albert Raymond, The end point compactification of manifolds ..

Edgar Reich and S. E. Warschawski, On canonical conformal maps of regions of arbitrary connectivity

Marvin Rosenblum, The absolute continuity of Toeplitz's matrices...

Lee Albert Rubel, Maximal means and Tauberian theorems . .

Helmut Heinrich Schaefer, Some spectral properties of positive linear operators

Jeremiah Milton Stark, Minimum problems in the theory of pseudo-conformal transformations and their application to estimation of the curvature of the invariant metric.

Robert Steinberg, The simplicity of certain groups ...

Hisahiro Tamano, On paracompactness. .

Angus E. Taylor, Mittag-Leffler expansions and spectral theory .

Marion Franklin Tinsley, Permanents of cyclic matrices ...... . 